\title{
Where the Blue Stragglers Roam: Searching for a Link Between Formation and Environment
}

\author{
Nathan Leigh $^{1}$, Alison Sills ${ }^{1}$ and Christian Knigge ${ }^{2}$ \\ ${ }^{1}$ Department of Physics and Astronomy, McMaster University, 1280 Main Street West, \\ Hamilton, ON, L8S 4M1, Canada \\ email: leighn@mcmaster.ca, asills@mcmaster.ca \\ ${ }^{2}$ School of Physics and Astronomy, Southampton University, Highfield, Southampton, SO17 \\ 1BJ, UK \\ email: christian@astro.soton.ac.uk
}

\begin{abstract}
Current observational evidence seems to indicate that blue stragglers are a dynamically created population, though exactly how the mechanism(s) of formation operates remains a mystery. We search for links between blue straggler formation and environment by considering only those stars found within one core radius of the cluster center. In so doing, we aim to isolate a sample that is representative of an approximately uniform cluster environment where, ideally, a single blue straggler formation mechanism is predominantly operating. Normalized blue straggler frequencies are found and apart from new anticorrelations with the central velocity dispersion and the half-mass relaxation time, we find no other statistically significant trends.

Concerns regarding the method of normalization used to calculate relative blue straggler frequencies are discussed, specifically whether the previously observed anticorrelation with total cluster mass (see Piotto et al. 2004) is a consequence of the normalization process. A new correlation between the observed number of blue stragglers in the core and the number predicted from single-single collisions alone is presented. This new link between formation and environment represents the first direct evidence that the blue straggler phenomenon has, at least in part, a collisional origin.
\end{abstract}

Keywords. blue stragglers - globular clusters: general

\section{Introduction}

Blue stragglers are stars that are brighter and bluer (hotter) than the main-sequence (MS) turn-off. Stars having their same mass have evolved off the MS and begun to ascend the giant branch (GB). First discovered by Sandage (1953) in the cluster M3, blue straggler stars (BSSs) are the quintessential example of how stellar evolution alone cannot adequately explain the presence of every stellar sub-population in the color-magnitude diagram (CMD). Specifically, BSSs are suspected to be indicative of the complex interplay between stellar evolution and stellar dynamics that is thought to occur in various cluster environments (see for example Ferraro et al. 2004; Sills et al. 2005).

There is an overall consensus that blue stragglers are the products of stellar mergers between two (or more) low mass MS stars, either through direct stellar collisions or the coalescence of binary systems (see for example Leonard (1989); Stryker 1993; Bailyn 1995). There is evidence to suggest that both formation mechanisms do operate, however the preferred mode of creation appears to depend on the cluster environment (see for example Mapelli et al. 2006). Observations from the Hubble Space Telescope (HST) indicate that BSSs are centrally concentrated in globular clusters (for instance Ferraro 
et al. 1999), though they have been found to have a bimodal radial distribution in clusters like M55 (Zaggia et al. 1997), M3 (Ferraro et al. 1997), and 47 Tuc (NGC 104) (Ferraro et al. 2004). In other words, BSS populations seem to exhibit elevated numbers in the cores of GCs, followed by a "zone of avoidance" at a few core radii from the cluster center, and then another rise in numbers towards the outskirts. This bimodal trend has been speculated to have arisen as a result of two separate formation mechanisms dominating in the cluster center and the peripheral regions, with collisions dominating in the latter and mass transfer in the former.

Recently, Piotto et al. 2004 examined the CMDs of 56 different GCs, comparing the BSS frequency to cluster properties like total absolute luminosity (mass), central density and the collision rate. Their relative frequencies were found by normalizing the number of BSSs to the horizontal branch (HB) or the red giant branch (RGB). They found that the most massive clusters had the lowest frequency of BSSs, and that there was a weak anticorrelation between BSS frequency and the cluster collisional parameter. They also showed that the BSS luminosity functions (BSLFs) of the most massive clusters had brighter peaks and reached brighter luminosities than did those of less massive clusters.

\section{Analysis}

We analyzed the CMDs of 57 GCs from Piotto et al.'s 2002 database in order to search for links between blue straggler formation and environment (see Leigh et al. 2007). By considering only those stars found within one core radius of the cluster center where the density is the highest, we chose a uniform dynamical environment where a single BSS formation mechanism is primarily occurring - namely collisions (Ferraro et al. 2004; Mapelli et al. 2006).

First, we consistently defined each stellar sub-population, applying the same set of boundary conditions to each cluster CMD in our sample. As such, it was necessary to define two reference points, namely the center of the mass of points that populate the main-sequence turn-off (MSTO) and the middle of the HB in the F555-plane. Two such landmarks proved necessary since the separation in the F555-plane between the MSTO and the HB often varies appreciably from cluster to cluster. From there, it was a simple matter of fitting the same set of boundary criteria to each cluster in our sample.

After confirming that the previously observed anticorrelation with total cluster mass (Piotto et al. 2004) also exists in the purely core population, we searched for other trends between normalized blue straggler frequencies and various cluster parameters. Apart from anticorrelations with the half-mass relaxation time and the central velocity dispersion both thought to stem from the fact that they are correlated with total cluster mass (see Spitzer 1987 and Djorgovski \& Meylan 1994 respectively) - no new statistically significant trends were found with any other cluster parameters, including two separate collisional parameters (Piotto et al. 2004; Pooley \& Hut 2006). This could reflect a complex interplay of influential parameters making it difficult to isolate any one connection, or that BSS formation does not really depend on the cluster environment.

We also generated cumulative BSLFs for every cluster in our sample, in addition to applying quadratic fits to each. Piotto et al. 2004 suggested that the resulting interior chemical profiles of collision products should differ from those of coalescence and that this could result in different observational signatures for each. If true and BSS formation does indeed depend on cluster mass, then one would expect the BSLF to similarly depend on cluster mass. However, the BSLFs of the most massive clusters showed no difference in peak magnitude with those off the least massive clusters. We also looked for trends between the various quadratic coefficients that accompanied our fits and the 
usual cluster parameters, but found none. These results suggest that either a single BSS formation mechanism is operating in all cluster cores, or that the products of collisions and coalescence are not observationally distinguishable, at least not long enough for the phenomenon to have much of a chance of being observed.

\section{Normalization}

As a result of finding a lack of trends between normalized BSS frequencies and the various cluster parameters considered, we became concerned that the normalization process could be obscuring trends. Indeed, the previously observed anticorrelation with total cluster mass appears to be, at least in part, a reflection of the correlation between the size of the stellar sub-population used for normalization - in our case the RGB - and total cluster mass. A plot of the total cluster $\mathrm{V}$ magnitude versus the logarithm of the inverse of the number of RGB stars shows that if one were to assume that each cluster in our sample has but a single blue straggler, normalizing the counts would still lead to a clear anticorrelation. We note that the effect is more pronounced in some clusters than others - that is, some of the BSS counts can fluctuate by a factor of quite a few (even getting close to a factor of ten at times) without greatly affecting their corresponding normalized BSS frequencies.

\section{Results}

A new correlation between observed core blue straggler counts and the number predicted from single-single collisions alone has been found, representing the first direct evidence that BSSs are, at least in part, formed via collisions. Fig. 1 shows a plot of the number of BSSs expected to populate the entire cluster core based on the application of a geometrical scaling factor (which proved necessary due to the varying degree of coverage exhibited by the HST field of view on a cluster-to-cluster basis) versus the number predicted from single-single collisions alone. Our predictions for the number of collisionally-produced BSSs comes from multiplying the average blue straggler lifetime $\tau_{B S S}$, taken to be 1.5 Gyrs (see Sandquist et al. 1997), by the total number of singlesingle collisions expected to occur in the core based on the derived timescale of Leonard (1989). Note that upon applying a density cut at $10^{4} \mathrm{M}_{\odot} \mathrm{pc}^{-3}$, a statistically significant correlation in the densest clusters becomes apparent. In other words, in clusters denser than this, there is good overall agreement between the observed number of BSSs and the number predicted from single-single collisions. In clusters less dense than this, however, relatively few BSSs are expected to be produced from single-single collisions and yet the observations show relatively populous blue straggler populations. This discrepancy implies that some other mechanism, possibly mass-transfer, is responsible for many BSSs in sparse cluster environments.

In dense cluster cores having a high single-binary (and binary-binary) encounter rate, binary systems that could undergo prolonged and unimpeded mass-transfer may continually be disrupted by single stars. This could account for the discrepancy seen in less dense cores wherein only a few BSSs are expected to be the products of single-single collisions since most binary systems undergoing mass-transfer in these clusters should remain uninterrupted. It seems that mass-transfer is responsible for many of the BSSs we see in low density cluster environments, specifically GC cores less dense than around $10^{4} \mathrm{M}_{\odot}$ $\mathrm{pc}^{-3}$. One has to wonder, however, why the agreement is so good in the densest clusters without having to factor in the inward migration of BSSs into the core from the cluster outskirts. If some of the core BSSs in those clusters less dense than $10^{4} \mathrm{M}_{\odot} \mathrm{pc}^{-3}$ are a 


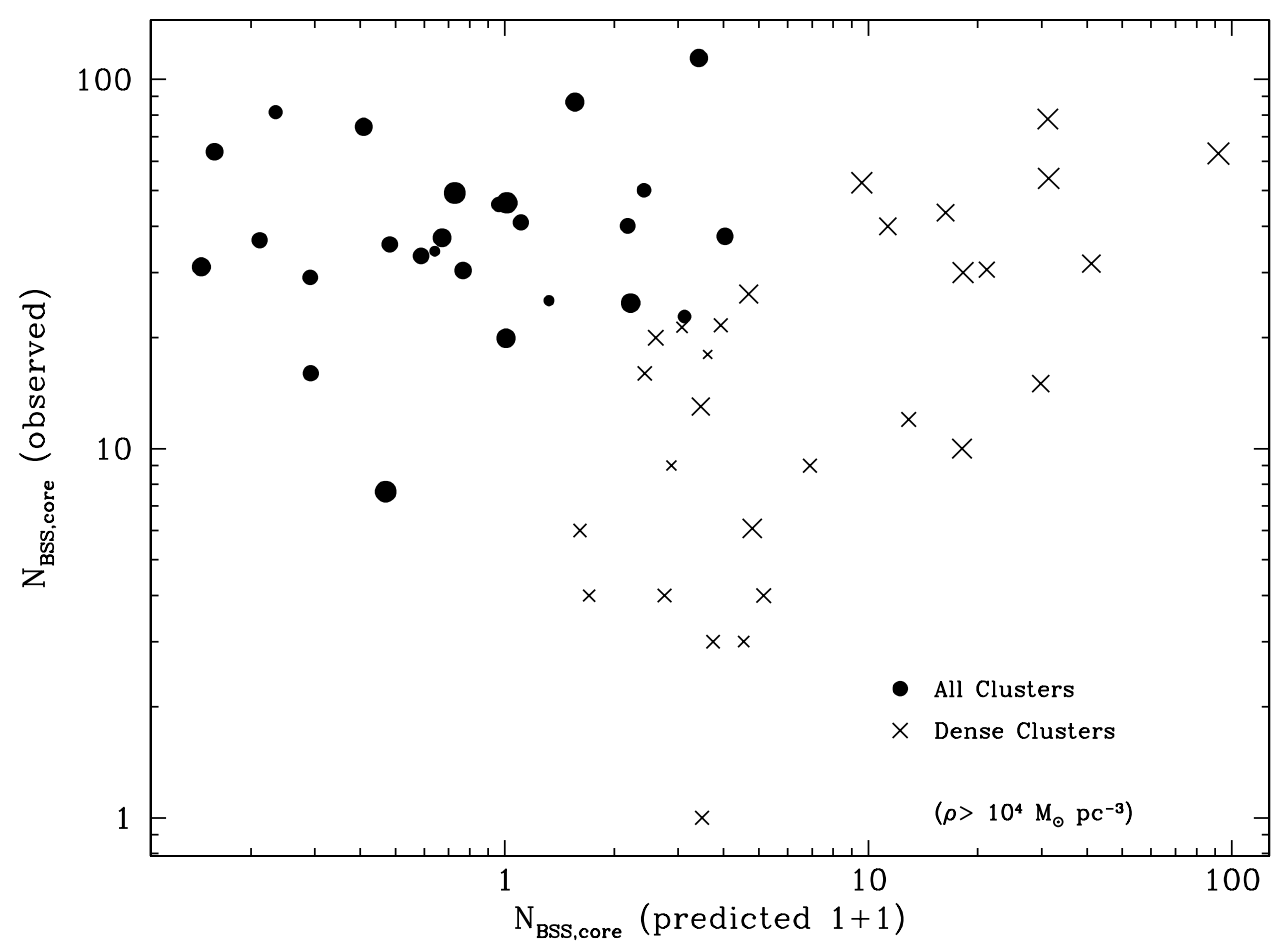

Figure 1. Plot of the number of core BSSs observed in Piotto et al's 2002 database after the geometrical scaling factor has been applied versus the number predicted in the core via single-single collisions alone. The filled circles represent clusters having a central density less than $10^{4} \mathrm{M}_{\odot} \mathrm{pc}^{-3}$, whereas the crosses represent clusters denser than this - note the correlation in the densest clusters. The size of the circles and crosses scales with total cluster mass such that the largest ones correspond to the most massive clusters.

result of the inward migration of primordial binaries (PBs) from the cluster outskirts, then why is this effect not seen in the densest clusters where single-single collisions alone can account for the observations? Perhaps most PBs migrate into the core within the first few Gyrs of cluster evolution and have hence evolved away from the blue straggler phase long before they can be observed.

\section{Conclusions}

We showed that normalized blue straggler frequencies do not appear to be correlated with any global cluster parameters. Consequently, a direct link between blue straggler formation and environment remains elusive, at least when considering BSS frequencies. Even upon generating BSLFs for every cluster in our sample, in addition to applying quadratic fits to each, no trends with any cluster parameters were apparent.

We grew suspicious that normalizing the data was obscuring trends. After considering non-normalized BSS numbers, we found a statistically significant correlation between the number of observed BSSs in the core and the number predicted from single-single collisions in clusters having a core density greater than $10^{4} \mathrm{M}_{\odot} \mathrm{pc}^{-3}$. In sparser cores, however, the agreement between our predictions and the observations is poor and we suspect that mass-transfer and coalescence are responsible for creating the majority of 
the BSSs therein. While more work still needs to be done, it certainly seems plausible that single-binary and binary-binary encounters could be inhibiting mass-transfer in dense cluster cores. With more numerous and better constrained estimates of GC core binary fractions, a great deal of light could be shed on how exactly these enigmatic stellar bodies are created.

\section{References}

Bailyn, C. D. 1995, ARAA 33, 133

De Angeli, F., Piotto, G., Cassisi, S., Busso, G., Racio-Blanco, A., Salaris, M., Aparicio, A., Rosenberg, A. 2005, AJ 130, 116

Djorgovski, S., \& Meylan, G. 1994, ApJ 108, 1292

Ferraro, F. R. et al. 1997, A\&SA 324, 915

Ferraro, F. R., Paltrinieri, B., Rood, R.T., Dorman, B. 1999, ApJ 522, 983

Ferraro, F. R., Sills, A., Rood, R. T., Paltrinieri, B., \& Buonanno, R. 2003, ApJ 588, 464

Ferraro, F. R., Beccari, G., Rood, R. T., Bellazzini, M., Sills, A., \& Sabbi, E. 2004, ApJ 603, 127

Harris, W. E. 1996, AJ 112, 1487

Knigge, C., Zurek, D. R., Shara, M., M., Long, K. S., \& Gilliland, R. L. 2003, ApJ 599, 1320

Knigge, C., Gilliland, R. L., Dieball, A., Zurek, D. R., Shara, M. M., \& Long, K. S. 2006, ApJ 641,281

Leigh, N. Sills, A. \& Knigge, C. 2007, ApJ 661, 210

Leonard, P. J. T. 1989, AJ, 98, 217

Mapelli, M., Sigurdsson, S., Ferraro, F. R., Colpi, M., Possenti, A. \& Lanzoni, B. 2006, MNRAS, 373,361

Piotto, G. et al. 2002, A\&BA 391, 945

Piotto, G. et al. 2004, ApJL 604, L109

Pooley, D. \& Hut, P. 2006, ApJ 646, 143

Pryor C. \& Meylan, G. 1993, in: S.G. Djorgovski \& G. Meylan (eds.) ASP Conf. Series, Structure and Dynamics of Globular Clusters (San Francisco: ASP), vol. 50, p. 357

Sandage, A. R. 1953, AJ, 58, 61

Sandquist, E. L., Bolte, M. \& Hernquist, L. 1997, ApJ, 477, 335

Sills, A. R. \& Bailyn, C. D. 1999, ApJ, 513, 428

Sills, A. R., Adams, T. \& Davies, M. B. 2005, MNRAS, 358, 716

Spitzer, L. 1987, Dynamical Evolution of Globular Clusters, (Princeton: Princeton University Press)

Stryker, L. L. 1993, PASP, 105, 1081

Zaggia, S. R., Piotto, G. \& Capaccioli, M. 1997, A\&A 327, 1004

Zinn, R. \& West, M. J. 1984, ApJS 55, 45 